\title{
Teoria das Restrições, Lean Manufacturing e Seis Sigma: limites e possibilidades de integração
}

\author{
Diego Augusto de Jesus Pacheco ${ }^{\text {a* }}$ \\ a*profdajp@gmail.com, UFRGS, Brasil
}

\begin{abstract}
Resumo
0 principal objetivo deste estudo foi analisar os pontos de convergência e as divergências entre a Teoria das Restrições, o Lean Manufacturing e o Seis Sigma quando usados de maneira integrada para a melhoria contínua operacional de sistemas produtivos. 0 eixo principal de discussão desse estudo foi buscar identificar na literatura características de exclusão e similaridades entre as três abordagens quando aplicadas de maneira integrada em sistemas produtivos. Para conduzir essa pesquisa, fez-se uma ampla pesquisa bibliográfica, entre 1995 e 2011, nas principais bases de dados nacionais e internacionais, em busca do estado da arte sobre o tema. Os resultados desse estudo sugerem que Teoria das Restrições, Lean Manufacturing e Seis Sigma possuem diversos elementos complementares que se sobrepõem aos pontos divergentes e que há um vasto campo de pesquisa a ser explorado sobre o tema. Como resultado, o presente estudo apresenta a análise crítica comparativa de 28 critérios relevantes à compreensão das três abordagens assim como uma agenda de pesquisa sobre o tema identificada a partir das lacunas encontradas.
\end{abstract}

Palavras-chave

Teoria das Restrições. Lean Manufacturing. Seis Sigma. Melhoria contínua.

\section{Introdução}

0 principal objetivo do presente artigo é investigar os fatores de convergências e divergências existentes entre o Lean Manufacturing, a Teoria das Restrições (Theory of Constraints - TOC) e o Seis Sigma quando usadas de forma combinada para a melhoria contínua de processos em ambientes de manufatura. A discussão fundamenta-se na medida em que o uso de abordagens focadas em melhoria contínua vem sendo feito por organizações, e, via de regra, tais abordagens já atingiram seu limite de desempenho frente à competitividade atual e à complexidade de alguns mercados competitivos atuais. Além disso, se faz necessário buscar elementos de outras abordagens para tornar mais robustas as atuais estratégias de melhoria contínua adotadas atualmente nas empresas brasileiras frente à competitividade global. Nesse sentido, alguns autores vêm estudando a combinação de abordagens para oferecer modelos integrados de melhoria contínua. Stamm et al. (2009) analisaram a evolução e diferenças fundamentais entre TQM, TPM, TOC, Lean e Seis Sigma, contrapondo essas abordagens com o modelo de produção fordista. Verificou-se que: i) Lean possui um paradigma de manufatura superior baseado na produção puxada em relação à empurrada; ii) é possível desenvolver modelos integrando Lean e as filosofias de gerenciamento da qualidade; iii) a combinação da TOC com outras abordagens indica resultados superiores em relação aos demais modelos. Em contrapartida, os estudos de Nave (2002), Pirasteh \& Fox (2011), Pirasteh \& Farah (2006) e Pirasteh \& Horn (2009), apesar de não terem o viés e rigor acadêmico, analisaram brevemente alguns aspectos entre as três abordagens, dentre eles foco, pressupostos, críticas e efeitos primários e secundários. Foram identificadas convergências entre as três abordagens e a sugestão de que o maior desafio para as organizações é escolher os pontos fortes de cada abordagem.

Assim sendo, é importante destacar que, a partir da revisão da literatura nas bases de dados pesquisadas, não se encontrou um estudo comparativo de rigor acadêmico, sob a lógica blind review, que tenha 
discutido em específico a TOC, Lean e Seis Sigma tendo como prisma de investigação os limites e possibilidades de integração visando à melhoria contínua operacional. Para constatar essa aparente lacuna na literatura em gestão de operações e na engenharia de produção, o presente estudo pesquisou publicações no período entre 1995 e 2011, nas seguintes bases de dados: Emerald, Springer Link, Scopus, Ebsco, Proquest, Scielo, IBTD, Periódicos Capes, UMI, ENEGEP e SIMPEP. A lacuna evidenciada a partir da falta de artigos científicos discutindo essas três tradicionais abordagens foi, portanto, uma das principais justificativas para o desenvolvimento dessa pesquisa. Ao fazer uma análise crítica comparativa entre tais abordagens, essa pesquisa também pretendeu objetivamente apresentar suas principais convergências e divergências, contribuindo para a tomada de decisão gerencial.

Dessa maneira, para conduzir a pesquisa, estruturou-se este artigo da seguinte forma: a seção 2 apresenta o referencial teórico sobre TOC, Lean e Seis Sigma; a seção 3 descreve e justifica os procedimentos metodológicos adotados nas etapas da pesquisa, coleta de dados e resultados iniciais; a seção 4 faz a análise comparativa aos pares entre TOC e Lean, TOC e Seis Sigma e Lean e Seis Sigma, visando identificar forças e fraquezas entre as abordagens. Essa seção é finalizada com a apresentação de um quadro síntese comparativo entre 28 critérios para compreensão conjunta das três abordagens; por fim, a seção 5 faz as conclusões e sugestões de trabalhos futuros despertadas a partir da revisão da literatura e da pesquisa como um todo.

\section{Referencial teórico}

\subsection{Teoria das Restrições (Theory of Constraints - TOC)}

A TOC foi desenvolvida pelo físico de notório conhecimento em sistemas Eliyahu M. Goldratt, durante os anos 80, e divulgada a partir do livro de negócios A Meta, de 1984. Entretanto, as origens da TOC estão relacionadas ao desenvolvimento de um software de programação da produção durante a década de 70, conhecido como Optmized Production Technology (OPT), também idealizado por Goldratt. Conforme os aperfeiçoamentos realizados no software, alguns conceitos inovadores de gestão da produção, como o TPC (Tambor-Pulmão-Corda), foram sendo formalizados na TOC. Segundo Souza (2005), existe na literatura uma confusão sobre a origem da TOC e é comum encontrar estudos apontando a OPT como sinônimo da abordagem para programação da produção, atualmente proposta pela TOC. Lacerda \& Rodrigues (2007) diferenciam o software OPT do pensamento OPT, ao afirmar que o pensamento OPT é a formalização de princípios que embasava o software OPT e que o livro $A$ Meta propunha popularizar tal pensamento e princípios.

Recentemente, pesquisas analisando a evolução da TOC têm sido realizadas. Gupta \& Boyd (2008) investigaram a amplitude da TOC fazendo uma análise com a gestão de operações e obtiveram os seguintes achados: (i) a TOC oferece um novo paradigma em gestão de operações que substitui um ultrapassado consenso de se buscar eficiência para alcançar a meta da empresa; e, assim, a busca da meta a partir de uma perspectiva global seria mais coerente para esse novo paradigma em gestão de operações; (ii) a TOC possui no seu escopo critérios de definição e enquadramento como uma teoria válida em gestão de operações; contudo, mais testes empíricos são necessários a fim de validar a TOC como uma teoria válida em operações. Então, a conclusão geral é que, devido ao aperfeiçoamento e evolução do escopo da TOC ao longo dos anos, esta começa a ser discutida e analisada na perspectiva de se tornar uma teoria válida no campo da gestão de operações. E isso implica na necessidade de sucessivas pesquisas relacionando-a com temas pertinentes e correlatos, como pretende, por exemplo, essa pesquisa ao discuti-la em ressonância com o Lean e o Seis Sigma já consolidados.

Avançando no entendimento atual sobre a TOC, Inman et al. (2009) ampliaram o modelo proposto por Boyd \& Gupta (2004) e, após analisar implementações da TOC, concluíram que: (i) quando completamente implementada (logística, processo de pensamento e indicadores de performance), a TOC é uma efetiva filosofia gerencial que resulta em resultados positivos como aumento do ganho, redução dos níveis de inventário e de despesa operacional, melhorando assim a performance organizacional; (ii) contrariando a ideia de que a orientação ao ganho melhora diretamente 0 desempenho organizacional conforme o estudo de Boyd \& Gupta (2004), Inman et al. (2009) concluíram que a relação entre a TOC e a performance organizacional é completamente mediada pelos resultados da TOC. Ou seja, a implementação da TOC não impacta diretamente na performance financeira e de mercado da firma conforme propunha Boyd \& Gupta (2004). A conclusão é que a implementação da TOC melhora os resultados, que por sua vez impactam positivamente na performance organizacional. Assim, o impacto da implementação da TOC é sentido primeiramente no nível operacional, indicando quais métricas relatadas para o sucesso da TOC poderiam focar sobre os resultados operacionais e organizacionais. Atualmente, 
o Processo de Pensamento da TOC se mostrou eficaz na tomada de decisão em pequenas empresas (Nonnemacher \& Pacheco, 2013).

\subsection{Lean Manufacturing}

Segundo Ghinato (1996), o sistema Toyota de produção (Toyota Production System - STP) tem sido, mais recentemente, referenciado como "sistema de produção enxuta". 0 termo Lean foi cunhado originalmente no livro $A$ Máquina que Mudou o Mundo, de Womack et al. (1992), como resultado de um amplo estudo sobre a indústria automobilística mundial realizado pelo MIT (Massachusetts Institute of Technology, EUA), no qual se evidenciaram as vantagens no uso do STP. 0 estudo evidenciou, entre outras questões, que o STP proporcionava expressivas diferenças em relação à produtividade, qualidade, desenvolvimento de produtos, e explicava o sucesso da indústria japonesa na época.

Segundo Ohno (1997), os pilares do STP ou o chamado espírito Toyota são dois: (i) a autonomação, também entendida como automação com toque humano, que consiste em dotar máquinas, equipamentos e pessoas da autonomia necessária de parar a produção sempre que uma condição preestabelecida, ou normal, for atingida (como a quantidade produzida) ou em condição anormal (em casos que há desvios no padrão de qualidade desejado); (ii) just in time, uma lógica de produção que tem como princípio gerar estoque em níveis estritamente necessários ao sistema, de forma a produzir no exato tempo apenas a quantidade necessária de produtos.

Segundo Shingo (1996), todos os sistemas de produção podem ser analisados sob duas perspectivas: função processo e função operação, sendo: (i) função processo: o fluxo do produto (materiais, tarefas, ideias) no tempo e no espaço, rompendo assim com o paradigma tradicional (just in case), que enxergava o processo como um conjunto de operações; (ii) função operação: o fluxo do sujeito de trabalho no tempo e no espaço, ou seja, os operadores e as máquinas. Nesse sentido, o autor propõe que o foco das ações de melhoria deve ser a função processo, ou seja, no fluxo da matéria-prima, o qual sofre forte impacto das esperas.

Como forma de explicar o Lean thinking, Liker \& Meier (2007) sugerem os 4Ps que explicariam os princípios da produção enxuta: (i) philosophy (filosofia): a base para o pensamento de longo prazo é a filosofia enxuta, onde os líderes veem a empresa como um veículo para agregar valor aos clientes, à sociedade, à comunidade e aos seus funcionários; (ii) process (processo): o princípio é que processos certos geram resultados certos; (iii) people \& partners (pessoas e parcerias): segundo esse princípio é fundamental o desenvolvimento de longo prazo, de pessoas e parceiros como modo de adição contínua e sistemática de valor aos clientes; e uma boa alternativa é agregar valor ao clientes desafiando seus funcionários e parceiros a crescer; (iv) problem solving (solução de problemas): a solução contínua da raiz dos problemas conduz à aprendizagem organizacional e à melhoria contínua. logo, com o intuito de melhor compreender o que é realmente o Lean, faz necessário trazer algumas definições presentes na literatura. Para Hopp \& Spearman (2004), Lean Production é um sistema integrado que realiza a produção de produtos e serviços usando o mínimo de estoques com baixos custos. Para Shah \& Ward (2007), Lean é um sistema sociotécnico integrado, cujo objetivo principal é eliminar o desperdício pela concomitante redução ou minimização da variabilidade em fornecedores, clientes ou dentro da empresa.

Recentemente, diversos autores ligados à academia têm discutido o que realmente é o Lean e quais são seus elementos. Pettersen (2009) realizou uma revisão sistemática das principais obras e autores sobre produção enxuta, e dentre as principais conclusões destacam-se as seguintes: (i) não existe na literatura um consenso sobre uma definição de Lean Production entre os principais autores do tema, tais como Ohno (1988), Shingo (1984), Monden (1998), Schonberger (1982), Feld (2001), Dennis (2002), Liker (2004), Bicheno (2004) e Womack \& Jones (2003) (apud Pettersen, 2009); e essa divergência pode causar confusão para acadêmicos e principalmente para praticantes que visam implementar tal conceito; (ii) uma organização não deveria aceitar uma variação aleatória do Lean, mas sim fazer escolhas e adaptar o conceito de Lean às suas próprias necessidades; e através desse processo de adaptação a organização irá se habilitar a elevar sua performance e aumentar as chances de sucesso da implementação; (iii) outra conclusão importante é que há uma concordância de que o Lean é muito mais que um conjunto de ferramentas, é sim uma filosofia; contudo, sobre os elementos do Lean não há uma concordância quanto ao conjunto de práticas e ferramentas que o formam. Pettersen (2009) identificou, por exemplo, que 100\% dos autores revisados concordam que fazem parte do Lean as práticas JIT (heijunka, produção puxada, produção no takt e sincronização dos processos), a redução de recursos (redução de lotes, eliminar perdas, setups, inventários, lead time), as estratégias de melhoria (kaizen e círculos de melhoria) e controle de defeitos (autonomação, poka yoke, inspeção 100\% e andons). Em contraposição, divergências foram 
encontradas, pois Supply Chain Management está presente em 78\% dos textos revisados, o controle estatístico da qualidade é discutido por 56\% dos autores e o TPM em 67\%.

\subsection{Seis Sigma}

A literatura tradicional sobre Seis Sigma, via de regra, remete suas origens às aplicações na Motorola na década de 1980. Entretanto, há diversas opiniões quanto aos seus verdadeiros mentores. Sharma (2003) afirma que foi desenvolvido por Mikel Harry em meados de 1980 para prover uma consistente abordagem focada na solução de problemas em negócios. Tal abordagem era baseada em dados para solucionar complexos problemas de negócios, identificando a causa raiz, a solução e o controle estatístico da solução. Pirasteh \& Fox (2011) identificaram como criador o nome de Bob Galvin, o presidente da empresa Motorola na época, e Bill Smith, um engenheiro.

Sob o ponto de vista estatístico, o sigma é uma medida de variabilidade intrínseca de um processo definido pelo desvio padrão e representado pela letra grega Sigma ( $\sigma)$. Sobre condições de normalidade, a medida Seis Sigma representa 2 partes por bilhão. Entretanto, considerando a flutuação de 1,5 sigma do processo em uma perspectiva de longo prazo, o processo tende a operar em uma taxa de 3,4 defeitos por milhão (PPM), o que efetivamente equivale a 4,5 sigmas em relação à média (Ehie \& Sheu, 2005). Assim, pelo conceito oriundo da Motorola, ainda que a média se desloque 1.5 sigmas em relação ao valor nominal, espera-se 3.4 defeitos por milhão de oportunidades. A Tabela 1 sinteticamente ilustra os principais valores adotados pela abordagem Seis Sigma, segundo Harry \& Schroeder (2000). De forma que, se o valor do desvio padrão é baixo, mais uniforme será o processo e menor variação existirá entre os resultados; e quanto menor for o desvio padrão, então melhor será o processo e menor será a possibilidade de falhas (Trad \& Maximiano, 2009).

Inicialmente o foco de aplicação do Seis Sigma se deu no contexto da manufatura. Todavia, com a maturidade da abordagem ao longo dos anos, o Seis Sigma vem ganhando força na área de serviços, saúde, alimentação e assim por diante. Segundo Santos \& Martins (2010), após o foco de gestão da qualidade se destacando na medição, em métodos quantitativos, equipe especializada e definição clara de metas de desempenho, o Seis Sigma, passou a ser usado num contexto mais amplo, sendo reconhecido como uma estratégia efetiva para melhorar o desempenho do negócio. Pacheco et al. (2013), por exemplo, integraram a técnica de mineração com as ferramentas Seis Sigma para melhorar os índices de qualidade na indústria.

Atualmente, com foco na oportunidade de ganhos financeiros tangíveis, as organizações aproximam o Seis Sigma das questões estratégicas para definir as diretrizes, sob uma perspectiva top-down. 0 estudo de Santos (2006) evidenciou que empresas que alinham os projetos Seis Sigma à estratégia corporativa possuem melhor desempenho em relação às que não o fazem. Além disso, outros fatores podem ser adicionados como críticos para o sucesso do Seis Sigma. 0 estudo de Trad \& Maximiano (2009) elencou os seguintes fatores: (i) liderança e participação da alta administração: deve ser ativa e com objetivos claramente traçados e comunicados; (ii) seleção de projetos: a escolha certa dos projetos alinhados à estratégia do negócio sob a óptica do cliente; (iii) recursos humanos: além do domínio técnico das abordagens quantitativas, competências como criatividade, colaboração, dedicação e comunicação são fundamentais; soma-se também a escolha correta da equipe.

Já a implementação propriamente dita do Seis Sigma envolve uma série de etapas focadas na melhoria contínua e os modelos adotados são o Define, Measure, Analyze, Improve e Control (DMAIC) e o DFSS (Design for Six Sigma), que adota o modelo DMADV (Define, Measure, Analyze, Design e Verify). 0 modelo DMAIC foi concebido e aperfeiçoado para aplicações em processos já existentes em ambiente de manufatura, processos e serviços. Enquanto que o modelo DMADV é adotado quando novas implantações de processos, produtos, serviços, etc. serão feitas ou

Tabela 1. Escala Sigma.

\begin{tabular}{|c|c|c|c|c|}
\hline Nível sigma & Nível da qualidade $(\%)$ & Taxa de erro $(\%)$ & $\begin{array}{l}\text { Defeitos por milhão de } \\
\text { oportunidades (DPMO) }\end{array}$ & $\begin{array}{l}\text { Custo da não qualidade } \\
\text { (\% do faturamento) }\end{array}$ \\
\hline $1 \sigma$ & 30,90 & 69,10 & 691,462 & Não se aplica \\
\hline $2 \sigma$ & 69,10 & 30,90 & 308,538 & Não se aplica \\
\hline $3 \sigma$ & 93,30 & 6,70 & 66,807 & 25 a 40 \\
\hline $4 \sigma$ & 99,38 & 0,62 & 6,21 & 15 a 25 \\
\hline $5 \sigma$ & 99,977 & 0,023 & 233 & 5 a 15 \\
\hline $6 \sigma$ & 99,99966 & 0,00034 & 3,4 & $<1$ \\
\hline
\end{tabular}


quando o nível sigma atual já está alto, em torno de cinco sigmas, conforme afirma Bendell (2006).

\section{Metodologia}

Pode-se dizer que essa pesquisa possui alguns aspectos de natureza básica, visto que irá tentar preencher uma lacuna na literatura da gestão de operações ao apresentar as convergências e divergências de integração entre TOC, Lean e Seis Sigma. Os resultados dessa discussão poderão gerar novos conhecimentos para os campos da engenharia de produção e da gestão de operações. Quanto aos objetivos, essa pesquisa pode ser definida como exploratória e descritiva. Exploratória, em primeiro lugar, porque a discussão exige atingir uma familiaridade com o objeto de pesquisa para, $a$ posteriori, realizar a articulação desses elementos com vistas a propor um quadro comparativo integrando a análise das abordagens. Em segundo lugar, porque, inicialmente, foi necessário elaborar o levantamento bibliográfico acerca das abordagens discutidas. É considerada descritiva, porque para responder à questão de pesquisa foram necessários identificar e estabelecer diversas relações de análise entre as abordagens analisadas, visando identificar suas convergências e divergências. Segundo Gil (2010, p. 29), a pesquisa bibliográfica é elaborada com base em material já publicado, seja impresso ou digital como: artigos, teses, revistas, dissertações etc. Praticamente toda pesquisa acadêmica requer em algum momento a realização de trabalho que pode ser caracterizado como pesquisa bibliográfica. Tanto é que, na maioria das teses e dissertações, uma seção é dedicada à revisão bibliográfica (Lima, 2004; Gil, 2010). Consoante com essa definição, essa pesquisa é classificada como bibliográfica, pois como pré-requisito para responder à questão de pesquisa foi necessário realizar a revisão teórica sobre a integração entre TOC, Lean e Seis Sigma.

A coleta de dados é um aspecto central em pesquisas qualitativas, sobretudo em pesquisas que fazem análise comparativa entre diferentes abordagens.
Acredita-se que na pesquisa científica, em geral, o primeiro passo é procurar trabalhos anteriores com preocupações similares (Biolchini et al., 2005 apud Silva, 2009). Assim sendo, uma relevante fonte de coleta de dados para esse estudo foi a revisão sistemática da literatura. Para Khan et al. (2001), a principal vantagem de usar um método de revisão sistemática é que fornece informação sobre a efetividade de intervenções ao identificar, avaliar e sumarizar os resultados de uma quantidade de dados não tratáveis de outra forma. Essa pesquisa usou como base os trabalhos de Silva (2009) e o estudo de Tranfield et al. (2003). As etapas do procedimento adotado para revisão da literatura foram: i) extrair palavras-chave a partir do problema de pesquisa: as palavras em inglês e em português escolhidas foram Lean Manufacturing, sistema toyota, produção enxuta, Lean Production, Seis Sigma, Six Sigma, Teoria das Restrições e Theory of Constraints; nesse caso, fez-se a combinação possivel aos pares das palavras-chave nos campos de busca do título e resumo das bases de dados, exemplo: Lean Manufacturing \& Six Sigma, Lean Manufacturing \& Theory of Constraints e assim por diante; ii) definir as bases de dados para busca de publicações: pesquisou-se em Emerald, Springer Link, Scopus, Ebsco, Proquest, Scielo-BR, ScieloInternacional, IBTD, Periódicos Capes, UMl, ENEGEP e SIMPEP; iii) definir horizonte de tempo de busca: pesquisou-se entre 1995 e 2011; iv) analisar títulos e resumos das publicações: foram analisados 836 trabalhos e a quantidade analisada em cada base de dados está detalhada na tabela do Apêndice 1; v) decidir entre a inclusão ou exclusão da publicação na pesquisa; vi) fazer análise, síntese e a inclusão das informações na pesquisa: essa discussão está consolidada na seção 4 deste estudo, onde os trabalhos com foco alinhados aos objetivos propostos no início da presente pesquisa foram inseridos. Cabe destacar, conforme apresentado na Tabela 2, que a pesquisa nas bases de dados mostra um número maior de publicações versando sobre Lean e Seis Sigma em relação ao número de publicações sobre TOC e Lean e TOC e Seis Sigma. A integração entre TOC e Seis

Tabela 2. Sintese do resultado da pesquisa bibliográfica.

\begin{tabular}{|c|c|c|c|c|c|c|c|c|c|c|c|c|c|c|c|c|c|}
\hline $\begin{array}{l}\text { Critérios de } \\
\text { busca/ano }\end{array}$ & 1995 & 1996 & 1997 & 1998 & 1999 & 2000 & 2001 & 2002 & 2003 & 2004 & 2005 & 2006 & 2007 & 2008 & 2009 & 2010 & 2011 \\
\hline $\begin{array}{l}\text { Lean e Seis } \\
\text { Sigma }\end{array}$ & 1 & & 1 & 1 & 3 & & 6 & 5 & 29 & 60 & 70 & 73 & 72 & 134 & 123 & 121 & 36 \\
\hline $\begin{array}{l}\text { Lean e } \\
\text { Teoria das } \\
\text { Restrições }\end{array}$ & & & & 3 & 1 & 3 & 1 & 3 & 3 & 5 & 6 & 7 & 7 & 9 & 13 & 7 & \\
\hline $\begin{array}{l}\text { Teoria das } \\
\text { Restrições e } \\
\text { Seis Sigma }\end{array}$ & 1 & & & 1 & 1 & & 1 & 3 & 1 & 3 & 4 & 3 & 3 & 2 & 7 & 3 & \\
\hline
\end{tabular}

Fonte: elaborado pelo autor. 
Sigma pareceu ser um tema recente na literatura, disponibilizando poucos estudos, tornando-se assim uma oportunidade de pesquisas futuras.

\section{Análises e discussões}

Uma vez apresentadas na seção 2 as principais definições sobre TOC, Lean e Seis Sigma individualmente, se faz necessário analisar criticamente as divergências e convergências entre tais abordagens, quando simultaneamente analisadas. São discutidos a seguir, então, estudos dos principais autores extraídos da pesquisa bibliográfica, que já investigaram a combinação dessas abordagens. Dentre os critérios usados para adotar o estudo pesquisado, foram priorizados os artigos de periódicos internacionais com fator de impacto maior que 1 , artigos com maior número de citações e artigos que discutem a integração entre as abordagens.

\subsection{Convergências e divergências entre Teoria das Restrições e Lean}

0 estudo de Dettmer (2001) indicou os seguintes pontos de similaridade entre as duas abordagens: possuem o objetivo comum de aumentar lucros, o valor é definido pelo cliente, o fator qualidade é essencial em ambas, preconizam a produção em lotes menores, visam o fluxo contínuo e o aumento da capacidade, buscam a minimização do inventário, e a participação da força de trabalho cumpre papel relevante no sucesso do desdobramento do método e das ferramentas. Alguns estudos de simulação computacional comparando JIT e TOC foram realizados em Miltenburg (1997), Chakravorty \& Atwater (1996), Cook (1994) e Watson \& Patti (2008). Miltenburg (1997) evidenciou que JIT opera com menor inventário e lead time, enquanto a TOC gera maior produtividade. Chakravorty \& Atwater (1996) concluíram que TOC é adequada para sistemas com variabilidade e downtimes (indisponibilidade de produzir) relativamente altos, enquanto JIT é melhor em sistema de variabilidade e downtimes baixos. Cook (1994) concluiu que a performance da TOC é melhor e que o JIT teria que eliminar virtualmente todas as variabilidades do sistema para tornar o desempenho semelhante ao da TOC. 0 survey realizado por Sale \& Inman (2003) mostrou que o uso combinado de JIT e TOC pode resultar em uma performance maior em relação ao uso individual das abordagens. Watson \& Patti (2008) concluíram que TOC é mais tolerante à variabilidade, tem menor lead time e precisa em média 50\% a menos de inventário que o JIT para a mesma produtividade. Um modelo de referência para realizar a gestão estratégica da capacidade produtiva da manufatura integrando TOC e indicadores de eficiência pode ser encontrado em Pacheco et al. (2012).

Já Antunes Junior (1998), em busca de um modelo geral, cita que as lógicas propostas pela TOC e da produção enxuta apresentam elevado grau de concordância à medida que propõem a visão do sistema produtivo como um todo. 0 autor discutiu convergências, divergências e pontos independentes que existem em uma abordagem e não estão presentes na outra. As principais convergências encontradas foram: (i) TOC e STP partem de uma visão de sistema aberto no que tange à concepção dos sistemas de produção; (ii) TOC e STP estão vinculados ao paradigma da melhoria nos processos; (iii) utilizam-se do método científico aplicando-o para a solução de diversos problemas ligados à administração da produção; (iv) partem de uma lógica dedutiva para desenvolver soluções técnicas específicas para a melhoria dos sistemas produtivos. É possível, a partir do conhecimento básico dos princípios que sustentam as duas teorias, gerar uma série de soluções particulares adaptadas aos casos específicos das empresas analisadas; (v) utilizaram-se e se utilizam da lógica dialética, em contraposição à lógica formal linear, para a busca de soluções originais dos problemas encontrados nos sistemas produtivos; (vi) privilegiam a gestão a partir dos subsistemas de melhorias, ou seja, dão uma ênfase prioritária ao subsistema de gestão das melhorias e da gestão da inovação. 0 objetivo central das duas propostas consiste em buscar um aprimoramento contínuo dos indicadores e na TOC isso é feito pelo acompanhamento dos indicadores globais e locais e no STP pela utilização das lógicas do custo-alvo e custo-kaizen; (vii) na TOC e no STP é dada uma prioridade máxima à lógica da sincronização da produção. Os esforços de melhorias propostos pela TOC e pelo STP estão diretamente relacionados com a óptica da melhoria contínua com foco centrado no aperfeiçoamento da sincronização da produção; (viii) os sistemas produtivos são visualizados enquanto uma cadeia ou corrente de eventos ou processos. Na TOC dá-se uma ênfase à gestão do elo mais fraco da corrente via os cinco passos da TOC, enquanto a gestão da cadeia no STP é feita via o kanban que, continuamente, mostra os elos fracos da cadeia (corrente). Nesse sentido, a decisão entre o balanceamento da capacidade dos recursos produtivos, a partir da lógica da TOC e dos gargalos em comparação com o balanceamento de fluxo da lógica Lean, aponta que o balanceamento da capacidade é mais eficaz. Uma discussão aprofundada sobre esse tema pode ser encontrada em Pacheco et al. (2013).

Já as principais convergências quanto à abordagem logística para Antunes Junior (1998) são: (i) têm em 
seu centro duas preocupações centrais: a necessidade da sincronização da produção e do estabelecimento de um processo sistemático de melhorias contínuas; (ii) possuem técnicas específicas para abordar a problemática da sincronização, na TOC a lógica do Tambor-Pulmão-Corda (TPC) e na produção enxuta 0 kanban; (iii) são preocupadas com a melhoria contínua dos sistemas produtivos. Na TOC isso aparece no passo 4 (elevar a capacidade das restrições) a partir da análise já feita no passo 1 da TOC (identificar as restrições). Já o STP é um sistema completamente voltado às melhorias, pois foi desenvolvido a partir da eliminação sistemática de perdas; (iv) há a possibilidade de uso sinergético da logística da Teoria das Restrições e dos subsistemas e técnicas do STP para implantar melhorias efetivas nas empresas, sustentam a importância de vencer a inércia para a implantação das novas ideias, fazem uma crítica sistemática à utilização da contabilidade de custos tradicional para efeitos de tomada de decisão, enfatizam a importância de vencer a inércia para a implantação das novas ideias, usam um embasamento científico comum através de relações do tipo efeito-causa-efeito e o pensamento dialético para a identificação, análise e solução de problemas.

E as principais convergências quanto à solução de problemas para Antunes Junior (1998) são: (i) utilização de um embasamento científico comum através de relações do tipo efeito-causa-efeito; (ii) utilização do pensamento dialético para a solução de problemas, ou seja, um pensamento que rejeita soluções de compromisso para a solução de problemas e postulam a necessidade de construir soluções passíveis de serem implantadas.

Já para Scheinkopf \& Moore (1998), os pontos de aproximação entre TOC e Lean são: (i) percepção de valor pela perspectiva do cliente: no Lean o valor é claramente definido pelo cliente e na TOC a percepção de valor do cliente é fator determinante para o aumento de ganho do produto; (ii) fluxo de valor: o Lean adota o termo fluxo de valor e a TOC o termo valor adicionado para explicitar que o valor percebido pelo cliente é definido por uma cadeia de interdependências entre a fábrica e os fornecedores; (iii) fluxo e produção puxada: oferecem técnicas para controlar fluxo usando o conceito de puxar a demanda do mercado. 0 Lean puxa sequencialmente, já que o recurso não deve produzir até receber o sinal (kanban) do recurso à jusante. Puxar é a essência do TPC para sincronizar o gargalo com a demanda do mercado e promover a liberação de material no sistema; iv) busca pela perfeição: segundo Goldratt \& Cox (1984), uma empresa só irá prosperar a partir de mudanças, a partir da melhoria contínua. Essa ideia está expressa no passo 5 do processo de focalização da TOC e na filosofia kaizen do Lean.

Antunes Junior (1998) também destacou pontos independentes na TOC, que não estão presentes no STP que seriam: i) classificação V-A-T de layouts, que permite analisar em profundidade os sistemas de produção, não somente do ponto de vista da sincronização da produção, mas também da priorização das técnicas de produção construídas a partir do STP; ii) a adoção de um conjunto de indicadores globais da fábrica (lucro líquido, retorno sobre o investimento e caixa) interligados com indicadores operacionais da TOC: ganho, despesas operacionais e inventários. 0 que possibilita verificar se uma dada decisão local está devidamente alinhada com o ótimo global do sistema; iii) o método da TOC não teria similar nos outros métodos quanto à busca sistemática e criativa de soluções, isso graças à técnica da evaporação das nuvens que formaliza o uso do método dialético nos métodos de solução dos problemas. Em contrapartida, os pontos independentes do STP, que não estão presentes na TOC, seriam: iv) os princípios básicos de construção dos sistemas enxutos e a consequência disso, ou seja, o conjunto de subsistemas e técnicas de engenharia de produção. E por fim as divergências e contradições seriam: (i) no STP parece estar claro o conteúdo da transformação ou mudança, sobretudo a partir dos elementos da sua estrutura, como: os subsistemas de pré-requisitos básicos (operação-padrão, troca rápida de ferramentas, layout), subsistema de quebra-zero (5S e TPM), subsistema de defeito-zero (capabilidade de máquinas, autonomação, poka-yoke). Enquanto que, na TOC, essa discussão não existe formalizada. Isso porque a TOC discute os meios e não o conteúdo, através do processo de pensamento, para formular as estratégias de transformação.

Segundo Dettmer (2001), TOC e Lean evoluíram para uma filosofia de visão sistêmica e sugere que modelo híbrido das duas abordagens é mais robusto, produtivo e de mais fácil implementação e o aspecto principal está na seleção dos elementos para o modelo. Dettmer (2001) sugere os seguintes pontos de congruência: são metodologias de sistemas, a melhoria contínua e o fluxo contínuo são essenciais, o fluxo de valor se estende para além da produção, a qualidade é essencial, produção em pequenos lotes, produção puxada (make-to-order ao invés de make-to-stock) e liberam capacidades escondidas Entretanto, Dettmer (2001) propõe que as maiores diferenças residem em dois aspectos: como cada um trata a variabilidade e a incerteza e na forma como tratam os custos. Enquanto Lean visa à redução de custos fixos e variáveis, na TOC a redução de custos é limitada, mas a geração de ganho não, não tornando a redução de custos um objetivo secundário. A TOC aceita a variabilidade e 
a instabilidade de demanda e das operações usando pulmões estratégicos (físicos, tempo, capacidade), enquanto que o Lean visa constantemente reduzir as variabilidades. De forma geral, Dettmer (2001) considera que existe uma sobreposição substancial entre o paradigma da mentalidade enxuta e da TOC, onde a TOC oferece uma estrutura para orientar os esforços Lean evitando as armadilhas de aplicá-los onde eles são desnecessários.

Antunes Junior (1998) também apresentou as principais convergências quanto ao sistema de performance. Ou seja, ambos criticam a utilização da contabilidade de custos tradicional para a tomada de decisão, o que implicou na necessidade de construção de novos sistemas de performance. No caso da TOC a lógica dos indicadores globais (lucro líquido, retorno sobre o investimento e caixa) e os indicadores operacionais (ganho, despesas operacionais e inventário); na Toyota foi proposto um sistema de medidas de performance cujo objetivo prioritário consiste em reduzir custos (com isso aumentando os lucros) e não para calcular custos. Esse sistema é baseado nos chamados custos-alvo e no custo-kaizen. Um aspecto central evidenciado como lacuna nos modelos investigados que integram TOC e Lean e deve ser considerado no aperfeiçoamento de novos modelos é o conceito das perdas originadas pela má definição do mix de produtos, definidos como “pergas” por Pergher et al. (2011).

\subsection{Convergências e divergências entre Teoria das Restrições e Seis Sigma}

O Seis Sigma identifica projetos orientados para redução de defeitos no processo e para melhorias operacionais. Contudo, não envolve integralmente os operadores e não possui uma visão sistêmica para entender como esses projetos irão afetar o desempenho global do sistema. Segundo Husby (2007), esse aspecto pode levar não só à priorização de projetos sem impactos financeiros para a empresa, como também na eliminação dos impactos positivos em outros processos. Como alternativa, Husby (2007) sugere que os cinco passos de focalização da TOC podem complementar essa lacuna. Entretanto, o autor ressalta que o processo de pensamento da TOC para análise e solução de problemas usa uma linguagem intelectual complexa, exigindo a condução por especialistas treinados e uma diferente abordagem para operadores e gerência.

Na visão de Jin et al. (2009), o foco do Seis Sigma é o cliente e o da TOC é a empresa e que, apesar de serem filosofias diferentes, ambas vêm sendo usados por diversas indústrias em processos de melhoria; porque, enquanto o Seis Sigma exige soluções em profundidade, a TOC pode revelar os gargalos e elevá-los. Para Nave (2002), a forma comum de integração entre TOC e Seis Sigma consiste em identificar a restrição da empresa e usar o Seis Sigma para reduzir sua variação ou resolver esse problema.

Segundo Jin et al. (2009), as principais vantagens da combinação das duas abordagens são: (i) a restrição é analisada, medida e controlada por um conjunto de ferramentas estatísticas ampliando a compreensão do problema e as decisões; (ii) o gargalo é o primeiro ponto a ser analisado, gerando assim aumento de ganho financeiro pela empresa e o projeto Seis Sigma não será escolhido por uma única área de negócios e sim pela visão global da TOC que vai gerar os resultados do projeto em todo sistema. Já as desvantagens para Jin et al. (2009) são: (i) nem sempre a redução de variação irá elevar a capacidade da restrição; (ii) quando a redução da variação elevar a taxa de produção do gargalo, os processos à jusante podem gerar maiores índices de rejeições, já que o foco foi colocado apenas no gargalo; (iii) a incerteza entre aplicar os princípios da TOC e depois o projeto Seis Sigma ou o contrário. 0 modelo integrando TOC e Seis Sigma proposto por Jin et al. (2009) pressupõe um ambiente com orçamento restrito para efetuar melhorias e a aplicação do Seis Sigma nos recursos após o gargalo para garantir a qualidade e eficiência do sistema. Esse modelo foi replicado em uma empresa de manufatura de motores com resultados finais satisfatórios.

Para Ehie \& Sheu (2005) há semelhanças entre os processos de melhoria do Seis Sigma (DMAIC) e da TOC (cinco passos de focalização). Os autores propuseram um modelo integrado onde a etapa inicial de identificação da restrição é mesma nas duas abordagens; a etapa seguinte segue a lógica da TOC para explorar a capacidade usando as fases medir e analisar do Seis Sigma como apoio; a próxima etapa também segue a lógica da TOC para explorar a capacidade usando a etapa Improve do Seis Sigma e suas ferramentas estatísticas para eliminar os problemas e as causas apontadas na etapa anterior; a etapa quatro usa o passo subordinar da TOC e o controlar do Seis Sigma para garantir que todas as ações tomadas anteriormente sejam aplicadas no sistema; na etapa cinco são realizados esforços para elevar a capacidade da restrição e a última etapa avalia a próxima restrição para evitar a inércia do sistema. Para aperfeiçoar o modelo os autores sugerem incorporar o processo de pensamento da TOC para compreender as interações causa-efeito no sistema bem como adicionar outras abordagens voltadas à melhoria contínua. 


\subsection{Convergências e divergências entre Seis Sigma e Lean}

Para Arnheiter \& Maleyeff (2005), Lean e Seis Sigma implementam uma cultura de melhoria contínua em todos os níveis dentro da empresa. E a vantagem do uso integrado reside na abordagem científica e quantitativa de qualidade fornecida pelo Seis Sigma, em relação às técnicas do Lean. Os projetos Seis Sigma focalizam seus esforços em reduzir a variação a partir da proposta padrão, o que pode levar a não focar nas exigências do cliente, mas apenas num exercício de redução de custos. Por isso sugere-se em paralelo adotar a visão de fluxo do Lean (Bendell, 2006). Para Harrison (2006), o uso das abordagens de forma isolada pode não ser eficaz, sob pena de criar duas subculturas dentro da empresa, competindo pelos mesmos recursos humanos e financeiros.

Há um limite de integração porque a estratégia utilizada para a melhoria depende do problema a ser resolvido, e, portanto, deve haver alinhamento entre as duas abordagens para obter resultados eficazes (Bañuelas \& Antony, 2004). Para Sharma (2003), o Seis Sigma deve ser utilizado para impulsionar a implementação dos esforços Lean. Para Bendell (2006), o equilíbrio reside na criação de valor sob o ponto de vista do cliente, de forma a focar o mercado e ao mesmo tempo reduzir a variação para níveis aceitáveis, diminuindo custos. Bendell (2006) ainda argumenta que os dois paradigmas são catalisadores da mudança e podem representar um instrumento poderoso ao alinhar os aspectos culturais do Lean com os projetos Seis Sigma. Existe um potencial enorme de uma abordagem sustentável de mudança organizacional e melhoria de processos integrando Lean e Seis Sigma (Bendell, 2006).

Para Snee (2010), Seis Sigma é normalmente usado para resolver problemas complexos para os quais a solução é desconhecida. É fundamental lembrar que o objetivo é obter as causas do baixo desempenho e não apenas centrar-se nos sintomas. Nesse caso a visão de fluxo do Lean contribui para o uso do Seis Sigma e sugere-se o uso simultâneo das abordagens. Snee (2010) enumerou oito características-chave que contribuem para o desempenho ao se aplicar sinergicamente Lean e Seis Sigma: criam resultados financeiros, ativam o envolvimento da alta liderança, usam uma abordagem disciplinada (DMAIC), projetos são finalizados rapidamente, definição clara de sucesso, infraestrutura humana criada (belts), foco nos clientes e nos processos e o uso de uma abordagem estatística.

Já para Montgomery (2010), projetos de melhoria Lean podem ser gerenciados usando o DMAIC. Montgomery (2010) defende o uso do Seis Sigma e do Lean como um modelo que resgata a filosofia de melhoria contínua e o sistema de conhecimento profundo proposto por Deming. Higgins (2005) diferencia os dois sistemas, argumentando que Seis Sigma é executado por poucos indivíduos específicos dentro de uma empresa, enquanto que no Lean a capacitação envolve todos os níveis da empresa para identificar e eliminar atividades sem valor agregado. Em contrapartida, Mika (2006) assume a posição crítica de que as duas abordagens são incompatíveis porque 0 Seis Sigma não pode ser adotado pelo trabalhador de nível médio da manufatura e argumenta que o Lean é acessivel para esses trabalhadores ao incentivar o trabalho em equipe através de equipes multifuncionais e grupos de melhoria.

Por outro lado, Arnheiter \& Maleyeff (2005) apontam os seguintes aspectos entre as abordagens: as empresas Lean devem adotar o uso de dados quantitativos para tomar decisões e uma abordagem mais científica para a qualidade dentro do sistema. Enquanto que empresas que usam Seis Sigma, precisam de uma abordagem mais ampla dos sistemas, considerando os efeitos do desperdício no sistema como um todo. Bendell (2006) cita que Lean e Seis Sigma se tornaram filosofias mal definidas, resultando na redução da eficácia, e muitas vezes as metodologias apresentadas são colocadas juntas sem uma explicação lógica, sem nenhuma base teórica ou explicação para a escolha das técnicas. Spector (2006) salienta que ao adotar o Lean e o Seis Sigma os profissionais podem encontrar um número grande de projetos com resultados insuficientes para a quantidade de tempo necessário para finalizá-los. Para Bendell (2006), é preciso fazer ajustes nas duas abordagens de forma que possam resolver efetivamente os problemas enfrentados por uma empresa, e a questão resume-se em como usá-las de maneira integrada. Seis Sigma complementa a filosofia Lean, fornecendo ferramentas e conhecimento para resolver problemas específicos que são identificados ao longo da jornada Lean (Bendell, 2006).

Para Pepper \& Spedding (2010) e Bendell (2006), as desconfianças sobre Lean e Seis Sigma devem-se à maneira míope de implementação. Por exemplo, a redução dos níveis de inventário não pode ser aplicada em ambientes de alta variabilidade, e por isso uma abordagem sistemática precisa ser adotada para otimizar todo o sistema e concentrar as estratégias certas nos lugares corretos. Lean e Seis Sigma devem ser vistos como a plataforma para o início da mudança cultural e operacional, levando à transformação total da cadeia de abastecimento. Para Bendell (2006), tais programas têm uma característica comum: eles focalizam no uso do pensamento do lado esquerdo do cérebro e isso pode ser tanto a grande força como a fraqueza. Ou seja, o autor percebe a falta do pensar 
com o lado direito do cérebro, relacionado com a criatividade e a inovação, e sugere uma abordagem holística integrada, semelhante às práticas de grupos de melhorias presentes nas origens do sistema Toyota de produção. Bendell (2006) cita que tal prática contribui significativamente na implementação do Seis Sigma e do Lean.

Bendell (2006) cita que uma deficiência fundamental do Seis Sigma e do Lean é a frequente falta de alinhamento com a política da empresa. Apesar da origem do Seis Sigma e do Lean ao defender apenas a satisfação do cliente, muitos projetos são escolhidos pelo custo baixo de implementação. Concentrando-se no controle e na redução da variação e do desperdício, o custo pode ser reduzido e a satisfação dos clientes pode ser aumentada (Bendell, 2006). Por outro lado, segundo Snee (2010), a natureza do problema é que define a escolha da abordagem e das ferramentas a serem usadas. Para alteração do processo ou da variação do processo é adequado usar o Seis Sigma, e nos casos de melhoria no fluxo de processo, de informações, de materiais ou redução da complexidade é indicado o uso do Lean. Contudo, para Snee (2010), ambas podem ser usadas para redução de perdas, de valor não agregado e de tempo de ciclo. Uma alternativa para combinar as duas metodologias consiste em subordinar uma abordagem deixando a outra como dominante. Uma série de grandes empresas que adotam programas Seis Sigma colocam Lean em um grupo de ferramentas adicionais dentro do programa Seis Sigma, porém o oposto não é tão comum (Bendell, 2006).

Pensando na construção de um novo modelo integrando Lean e Seis Sigma, segundo Pepper \& Spedding (2010) os principais fatores a serem analisados são: o modelo deve ser estratégico e focado em processos, o qual também deverá ter um balanceamento entre as duas filosofias aproveitando as reconhecidas vantagens de ambas, deverá ter equilíbrio entre a complexidade e sua sustentabilidade, e esse modelo deve ser estruturado sobre o tipo de problema ocorrido. Nessa proposta, Lean reforça a filosofia da estrutura e fornece direção estratégica para a melhoria, orientando a dinâmica geral do sistema e informando o estado atual das operações. Lean identifica as áreas fundamentais para a melhoria. Após serem identificados os pontos críticos, usam-se projetos Seis Sigma para focar na melhoria e conduzir o sistema para o estado futuro desejado.

A partir das reflexões anteriores constata-se, em suma, que: (i) as duas abordagens são predominantemente complementares e é viável pensar-se na integração entre as duas abordagens; (ii) na integração, o gerenciamento de projetos e a estratégia corporativa precisam estar alinhados, para evitar que haja sistemas separados entre a abordagem Lean e Seis Sigma; (iii) percebeu-se também que, se o Lean é implementado individualmente, faltam ferramentas específicas para alavancar seu pleno potencial conforme a complexidade do problema em análise. Da mesma maneira, se um projeto Seis Sigma é aplicado sem a visão sistêmica do Lean, o foco no fluxo global é esquecido e o desempenho do projeto de melhoria é comprometido.

Assim sendo, de forma a atender os principais objetivos dessa pesquisa de analisar os pontos de convergência e divergências entre as três abordagens sobre o prisma da melhoria contínua e ampliar o entendimento sobre seus os princípios fundamentais, elaborou-se o Tabela 3. A partir da revisão da literatura e das discussões anteriormente realizadas é apresentada uma síntese da análise crítica comparativa entre as três abordagens, a partir de 28 critérios de análise resultantes da revisão da literatura, extraídos a partir das reflexões geradas durante a realização da pesquisa e a partir, sobretudo, dos estudos de Antunes Junior (1998), Dettmer (2001), Scheinkopf \& Moore (1998), Husby (2007), Arnheiter \& Maleyeff (2005), Nave (2002), Stamm et al. (2009) e Pirasteh \& Fox (2011). A partir do resultado da discussão conduzida na seção anterior, alguns critérios relatados foram ampliados, modificados e outros diretamente relacionados. Foi considerado, a priori, que tais critérios são os fundamentais para o entendimento comparativos entre as abordagens, contribuindo para o entendimento acadêmico e para a tomada de decisão gerencial de integração. Contudo, é factível que tal relação de critérios de análise possa ser ampliada em futuras pesquisas que continuem a aprofundar o presente debate.

\section{Considerações finais e trabalhos futuros}

Este trabalho buscou analisar os pontos de convergência e de exclusão existentes entre Teoria das Restrições, Lean Manufacturing e Seis Sigma quando usados visando a melhoria contínua de processos em sistemas de manufatura. A discussão apresentada também tentou contribuir para o melhor entendimento sobre os princípios fundamentais de tais metodologias, realizando uma análise comparativa de aspectos considerados críticos. Após as análises realizadas, verificou-se que os objetivos de analisar os pontos de convergência e de exclusão existentes entre as três abordagens e contribuir para o melhor entendimento sobre seus princípios fundamentais foram atendidos. Constatou-se, de forma geral, que há mais pontos de sobreposição do que aspectos de exclusão entre as três abordagens e que é viável 


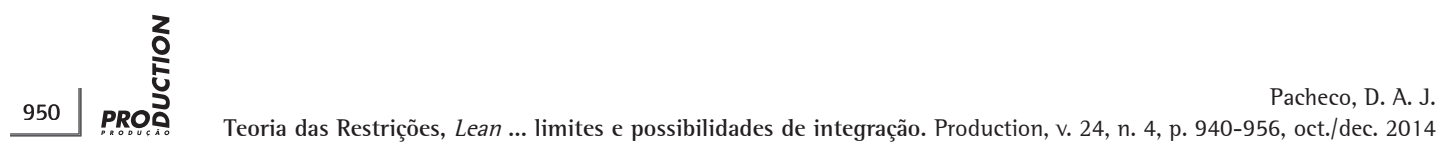

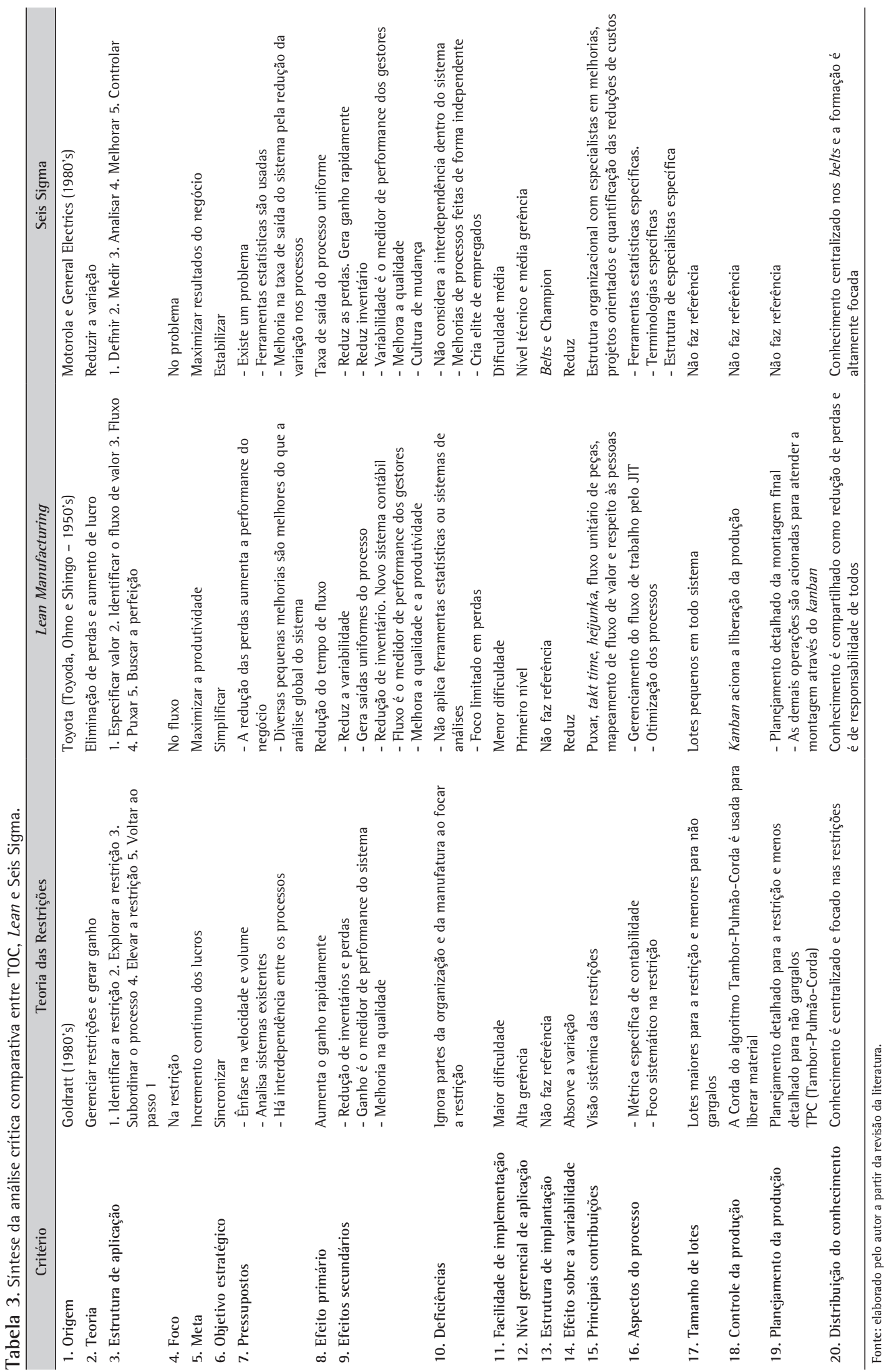




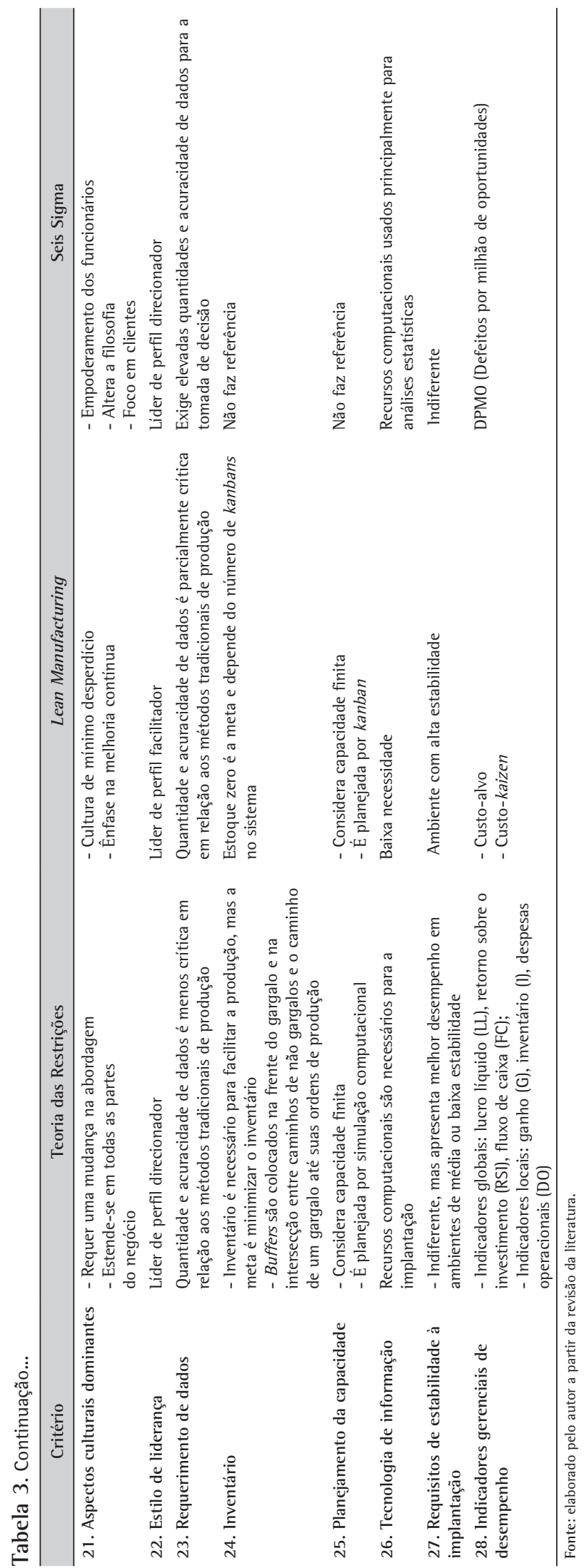


pensar na construção de um modelo integrado voltado para melhoria contínua de processos que aumente a competitividade tornando mais robustas as atuais estratégias, sobretudo das empresas brasileiras.

Entretanto, os resultados dessa pesquisa permitiram evidenciar que há fatores críticos que devem ser considerados no uso combinado ou ainda na construção de modelos integrando as três abordagens, sem os quais o desenvolvimento de um modelo holístico perde robustez. Dentre os principais fatores críticos, destaca-se que a discussão realizada na literatura ainda não apresenta uma definição clara sobre os seguintes aspectos: i) como escolher os elementos, práticas e ferramentas corretas de cada abordagem de acordo com a real necessidade da organização? ii) a empresa deve precisamente definir qual é sua prioridade: reduzir variabilidade? Reduzir perdas e melhorar o fluxo? Ou remover as restrições? iii) o correto diagnóstico sobre a cultura, objetivos, fraquezas e forças da organização também deve ser um aspecto considerado na integração das três abordagens e evidenciou-se a falta de pesquisas com esse foco; iv) outro fator crítico a ser considerado na integração das abordagens é a quebra de alguns usuais modelos mentais comuns nas empresas, tais como: 0 não envolvimento efetivo dos colaboradores do nível operacional, que é uma característica da cultura de implementação da TOC e do Seis Sigma; v) da mesma maneira, os princípios para a construção do modelo integrando tais abordagens devem obrigatoriamente estar alinhados com a estratégia da empresa e seus objetivos. Para empresas industriais de manufatura intensiva, um ponto de partida de alinhamento das três abordagens com a estratégia de produção, pode ser visto em Pacheco (2012).

Num âmbito geral, e sobretudo a partir da síntese do Tabela 3, os resultados do presente estudo permitiram verificar que a Teoria das Restrições, o Lean Manufacturing e o Seis Sigma possuem aspectos complementares que se sobrepõem aos pontos de exclusão e que há um vasto campo aberto para pesquisas sobre o tema. Assim sendo, com o propósito de avançar na discussão e no entendimento profundo das inter-relações entre as abordagens ou ainda avaliando a contribuição de outras abordagens, emerge finalmente além dos aspectos anteriormente citados a seguinte agenda de pesquisa para continuidade desse foco de investigação: i) quais indicadores devem ser usados para medir o desempenho do modelo integrando as três abordagens e como estruturá-los nos níveis da organização? ii) qual sistema de gerenciamento contábil deve ser usado na empresa para suportar o uso simultâneo das três abordagens nas operações da empresa? iii) como escolher qual será a cultura dominante da empresa e como construí-la, pressupondo que as três abordagens irão coexistir? iv) e, por fim, uma oportunidade de pesquisa de ordem prática seria investigar qual técnica de programação da produção é mais vantajosa (kanban ou TPC) e em quais ambientes deve-se implantar esta ou aquela.

\section{Referências}

Antunes Junior, J. A. V. (1998). Em direção a uma teoria geral do processo na administração da produção: uma discussão sobre a possibilidade de unificação da Teoria das Restrições e a teoria que sustenta a construção dos sistemas de produção com estoque zero (Tese de doutorado). Escola de Administração, Universidade Federal do Rio Grande do Sul, Porto Alegre.

Arnheiter, E. D., \& Maleyeff, J. (2005). The integration of Lean management and Six Sigma. TQM Magazine, 17(1), 5-18. http://dx.doi.org/10.1108/09544780510573020

Bañuelas, R., \& Antony, J. (2004). Six Sigma or design for Six Sigma? TQM Magazine, 16(4), 250-263. http://dx.doi. org/10.1108/09544780410541909

Bendell, T. (2006). A review and comparison of Six Sigma and the Lean organization. TOM Magazine, 18(3), 255-62. http://dx.doi.org/10.1108/09544780610659989

Boyd, L., \& Gupta, M. (2004). Constraints management: what is the theory? International Journal of Operations \& Production Management, 24(4), 370-371. http://dx.doi. org/10.1108/01443570410524631

Chakravorty, S. S., \& Atwater, B. J. (1996). A comparative study of line design approaches for serial production systems. International Journal of Operations e Production Management, 16(6), 91-108. http://dx.doi. org/10.1108/01443579610119117

Cook, D. P. (1994). A simulation comparison of traditional, JIT and TOC manufacturing Systems in a ?ow shop with bottlenecks. Production and Inventory Management Journal, 35, 73-78.

Dettmer, W. (2001). Beyond Lean Manufacturing: Combining Lean and the Theory of Constraints for Higher Performance. Port Angeles: Goal System International. Retrieved from http://www.goalsys.com/books/ documents/TOCandLeanPaperrev.1.pdf.

Ehie, 1., \& Sheu, J. (2005). Integrating Six Sigma and Theory of Constraints for continuous improvement: a case study. Journal of Manufacturing Technology Management, 16(5), 542-553. http://dx.doi. org/10.1108/17410380510600518

Ghinato, P. (1996). Sistema Toyota de produção: mais do que simplesmente Just-In-Time. Caxias do Sul: Ed. Universidade de Caxias do Sul.

Gil, A. C. (2010). Como elaborar projetos de pesquisa. São Paulo: Atlas.

Goldratt, E. M., \& Cox, J. F. (1984). A Meta. São Paulo: Nobel.

Gupta, M. C., \& Boyd, L. H. (2008). Theory of Constraints: A Theory for Operations Management. International Journal of Operations and Production Management, 28, 9-10. http://dx.doi.org/10.1108/01443570810903122

Harrison, J. (2006). Six Sigma vs. Lean manufacturing: Which is right for your company? Foundry Management \& Technology, 13(7), 31-32.

Harry, M., \& Schroeder, R. (2000). Six Sigma: the breakthrough management strategy revolutionizing the world's top corporations. New York: Doubleday. 
Higgins, K. T. (2005). Lean builds steam, Food Engineering: The Magazine for Operations and Manufacturing Management. Retrieved from http://www.foodengineeringmag.com/ articles/Lean-builds-steam.

Hopp, W. J., \& Spearman, M. L. (2004). To pull or not to pull: what is the question? Manufacturing \& Service Operations Management, 6(2), 133-148. http://dx.doi. org/10.1287/msom.1030.0028

Husby, P. (2007). Competition or Complement: Six Sigma and T0C. Material Handling Management, p. 51-55.

Inman, R. A., Sale, M. L., \& Green Junior, K. W. (2009). Analysis of the relationships among TOC use, TOC outcomes, and organizational performance. International Journal of Operations e Production Management, 29(4), 341-356. http://dx.doi.org/10.1108/01443570910945819

Jin, K. J., Hyder, A. R., Elkassabgi, Y., Zhou, H., \& Herrera, A. (2009). Integrating the Theory of Constraints and Six Sigma in Manufacturing Process Improvement. Proceedings of World Academy of Science, Engineering and Technology, 37.

Khan, K. S., Popay, J., \& Kleijnen, J. (2001). Development of a review protocol. In K. S. Khan, G. Ter Riet, J. Glanville, A. J. Sowden, J. Kleijnen (Eds.), Undertaking Systematic Reviews Of Research On Effectiveness (2nd ed, pp. 1931). New York: University of New York.

Lacerda, D., \& Rodrigues, L. H. (2007). Compreensão, aprendizagem e Ação: A abordagem do Processo de Pensamento da Teoria das Restrições. Resende: SEGeT.

Liker, J., \& Meier, D. (2007). Modelo Toyota - Manual de Aplicação: Um Guia Prático Para a Implementação dos 4Ps da Toyota. Porto Alegre: Bookman.

Lima, M. C. (2004). Monografia: a engenharia da produção acadêmica. São Paulo: Saraiva.

Mika, G. (2006). Six Sigma isn't Lean. Manufacturing Engineering, 137(1).

Miltenburg, J. (1997). Comparing JIT, MRP and TOC, and embedding TOC into MRP. International Journal of Production Research, 35(4), 1147-1169. http://dx.doi. org/10.1080/002075497195597

Montgomery, D. C. (2010). A modern framework for achieving enterprise excellence International. Journal of Lean Six Sigma, 1(1), 56-65. http://dx.doi. org/10.1108/20401461011033167

Nave, D. (2002). How to compare Six Sigma, Lean and the Theory of Constraints. Quality Progress, 73-79.

Nonnemacher, G. L., \& Pacheco, D. A. J. (2013). Impacto do Processo de Pensamento da Teoria das Restrições na tomada de decisão em pequenas empresas. Espacios (Caracas), 34, 1-12.

Ohno, T. (1997). O Sistema Toyota de Produção: além da produção em larga escala. Porto Alegre: Bokmann.

Pacheco, D. A. J. (2012). Integrando a Estratégia de Produção com a Teoria das Restrições, Lean e Seis Sigma: uma abordagem metodológica (Dissertação de mestrado). Universidade do Vale do Rio dos Sinos, São Leopoldo.

Pacheco, D. A., Antunes Júnior, J. A. V., Lacerda, D. P., Goldmeyer, D. B., \& Von Gilsa, C. (2012). Modelo de gerenciamento de capacidade produtiva: integrando Teoria das Restrições e o índice de rendimento operacional global (IROG). Produção Online, 12(3), 806-826.

Pacheco, D. A. J., Lacerda, D. P., Corcini Neto, S. L. H., \& Jung, C. F. (2013). Balanceamento de fluxo ou balanceamento de capacidade: análise e proposições sistêmicas. Gestão \& Produção. In press.

Pacheco, D. A. J., Machado, L., Jung, C. F., \& Caten, C. S. T. (2013). Investigando o uso da mineração de dados nos processos de gestão da qualidade total: um estudo de caso na indústria. Espacios (Caracas), 34, 1-11.

Pepper, M. P. J., \& Spedding, T. A. (2010). The evolution of Lean Six Sigma. International Journal of Quality e Reliability Management, 27(2), 138-155. http://dx.doi. org/10.1108/02656711011014276

Pergher, l., Rodrigues, L. H., \& Lacerda, D. P. (2011). Discussão teórica sobre o conceito de perdas do Sistema Toyota de Produção: inserindo a lógica do ganho da Teoria das Restrições. Gestão \& Produção, 18(4), 673-686. http:// dx.doi.org/10.1590/S0104-530X2011000400001

Pettersen, J. (2009). Defining Lean production: some conceptual and practical issues. TQM Journal, 21(2), 127142. http://dx.doi.org/10.1108/17542730910938137

Pirasteh, R. M., \& Farah, K. S. (2006). Continuous improvement trio. APICS Magazine, 31-33.

Pirasteh, R. M., \& Horn, S. (2009). The many sides of TLS. APICS Magazine, 40-43.

Pirasteh, R. M., \& Fox, R. E. (2011). Profitability with no boundaries. Quality press.

Sale, M. L., \& Inman, R. A. (2003). Survey-based comparison of performance and change in performance of firms using traditional manufacturing, JIT and TOC. International Journal of Production Research, 41(4), 829-844. http:// dx.doi.org/10.1080/0020754031000065520

Santos, A. B. (2006). Modelo de referência para estruturar o programa de qualidade Seis Sigma: proposta e avaliação (Tese de doutorado). Universidade Federal de São Carlos, São Carlos.

Santos, A. B., \& Martins, M. F. (2010). Contribuições do Seis Sigma: estudos de caso em multinacionais. Produção, 20(1), 42-53. http://dx.doi.org/10.1590/ S0103-65132010005000003

Scheinkopf, L., \& Moore, R. (1998). Theory of Constraints and Lean Manufacturing; Friend or Foes? Chesapeake Consulting.

Shah, R., \& Ward, P. T. (2007). Defining and developing measures of Lean production. Journal of Operations Management, 25(4), 785-805. http://dx.doi.org/10.1016/ S0272-6963(02)00108-0

Sharma, U. (2003). Implementing Lean principles with the Six Sigma advantage: how a battery company realized significant improvements. Journal of organizational Excellence, Summer.

Shingo, S. (1996). O Sistema Toyota de Produção: Do ponto de vista da engenharia de produção. Bookman.

Silva, É. R. P. (2009). Método para revisão e mapeamento sistemático da literatura (DEl-POLI/UFRJ) (Trabalho de Conclusão de Curso de Graduação). Universidade Federal do Rio de Janeiro, Rio de Janeiro.

Snee, R. D. (2010). Lean Six Sigma: getting better all the time. International Journal of Lean Six Sigma, 1(1), 9-29. http://dx.doi.org/10.1108/20401461011033130

Souza, F. B. (2005). Do OPT à Teoria das Restrições: mitos e avanços. Produção, 15(2), 184-197. http://dx.doi. org/10.1590/S0103-65132005000200005 
Spector, R. E. (2006). How constraints management enhances Lean and Six Sigma. Supply Chain Management Review, 42-46.

Stamm, M. L., Neitzert, T. R., \& Darius, P. K. (2009). TQM, TPM, TOC, Lean and Six Sigma -Evolution of manufacturing methodologies under the paradigm shift from Taylorism/Fordism to Toyotism? In Proceedings International Annual Euroma Conference, Gothenburg.

Trad, S., \& Maximiano, A. C. A. (2009). Seis Sigma: Fatores Críticos de Sucesso para sua Implantação. RAC, 13(4, art. 7), 647-662. http://dx.doi.org/10.1590/S141565552009000400008

Tranfield, D., Denyer, D., \& Palminder, S. (2003). Towards a methodology for developing evidence-informed management knowledge by means of systematic review. British Journal of Management, 14, 207-222. http:// dx.doi.org/10.1111/1467-8551.00375
Watson, J. K., \& Patti, A. (2008). A comparison of JIT and TOC buffering philosophies on system performance with unplanned machine downtime. International Journal of Production Research, 46(7), 1869-1885. http://dx.doi. org/10.1080/00207540600972943

Womack, J. P., Jones, D. T., \& Ross, D. (1992). A máquina que mudou o mundo. Rio de Janeiro: Campus.

\section{Agradecimentos}

0 autor da presente pesquisa agradece aos revisores pelas importantes contribuições durante o processo de avaliação do artigo, as quais contribuíram sobremaneira para melhorar a versão final desta pesquisa. Esta pesquisa foi parcialmente financiada pela Capes/CNPq.

\title{
Theory of Constraints, Lean Manufacturing and Six Sigma: limits to and possibilities for integration
}

\begin{abstract}
The objective of this study was to analyze points of convergence and divergence between the Theory of Constraints, Lean Manufacturing and Six Sigma, when used in an integrated manner for the continuous improvement of existing productive systems. The study's main focus was to search the literature and identify the characteristics of exclusion and the similarities between the three approaches when applied in an integrated manner to productive systems. A broad literature search encompassing the period 1995-2011 was conducted on the main national and international databases, seeking to identify the topic's state of the art research. The results of this study suggest that the Theory of Constraints, Lean Manufacturing and Six Sigma have many complementary elements that overlap their divergent points and that a vast field of research exists on this issue for future exploration. As a result, the study presents a critical analysis of 28 comparative criteria, relevant to the understanding of the three approaches, and concludes with a research agenda on the topic, based on identified gaps.
\end{abstract}

\section{Keywords}

Theory of Constraints. Lean Manufacturing. Six Sigma. Continuous improvement. 
Pacheco, D. A. J.

Teoria das Restrições, Lean ... limites e possibilidades de integração. Production, v. 24, n. 4, p. 940-956, oct./dec. 2014

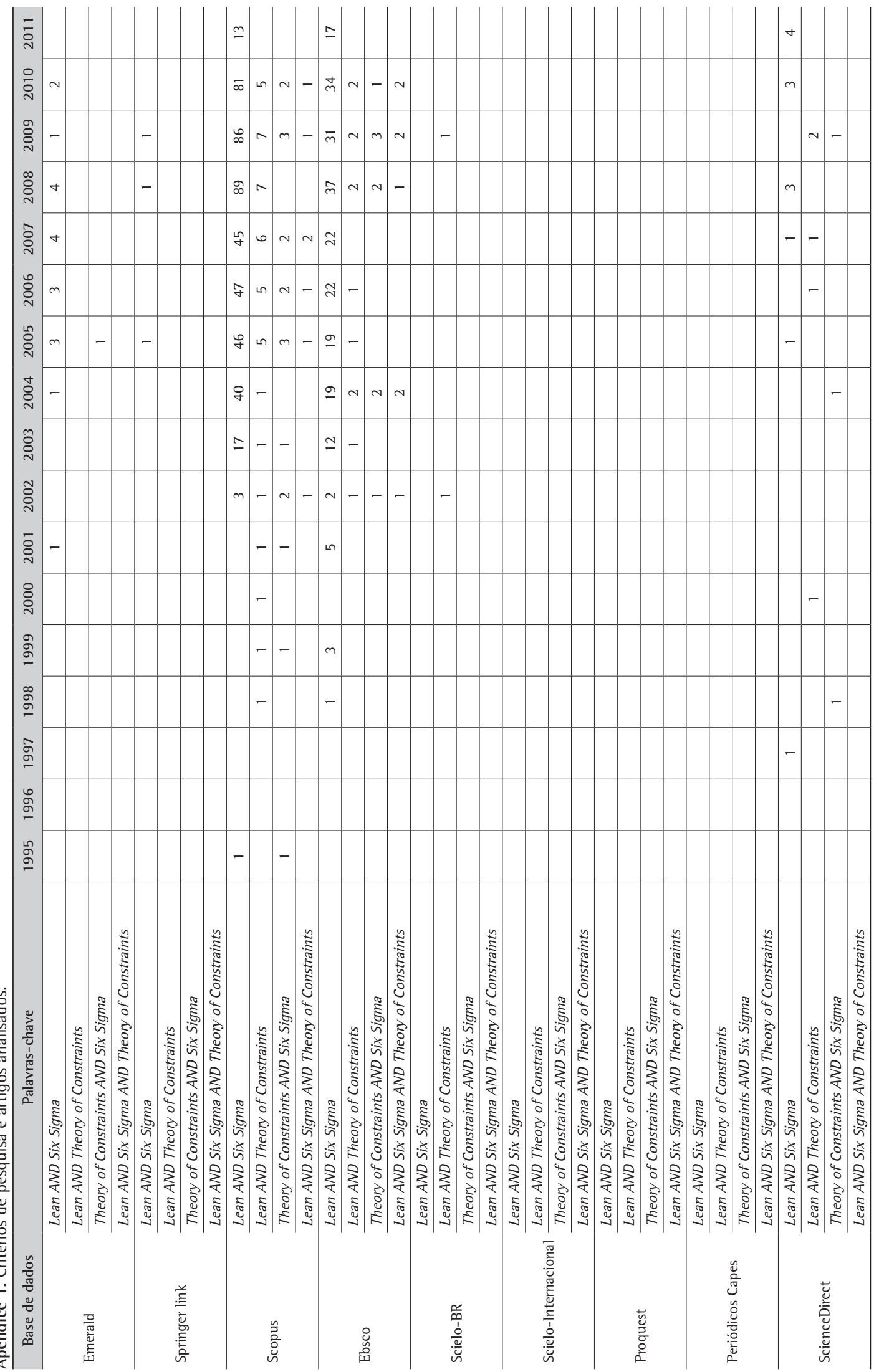




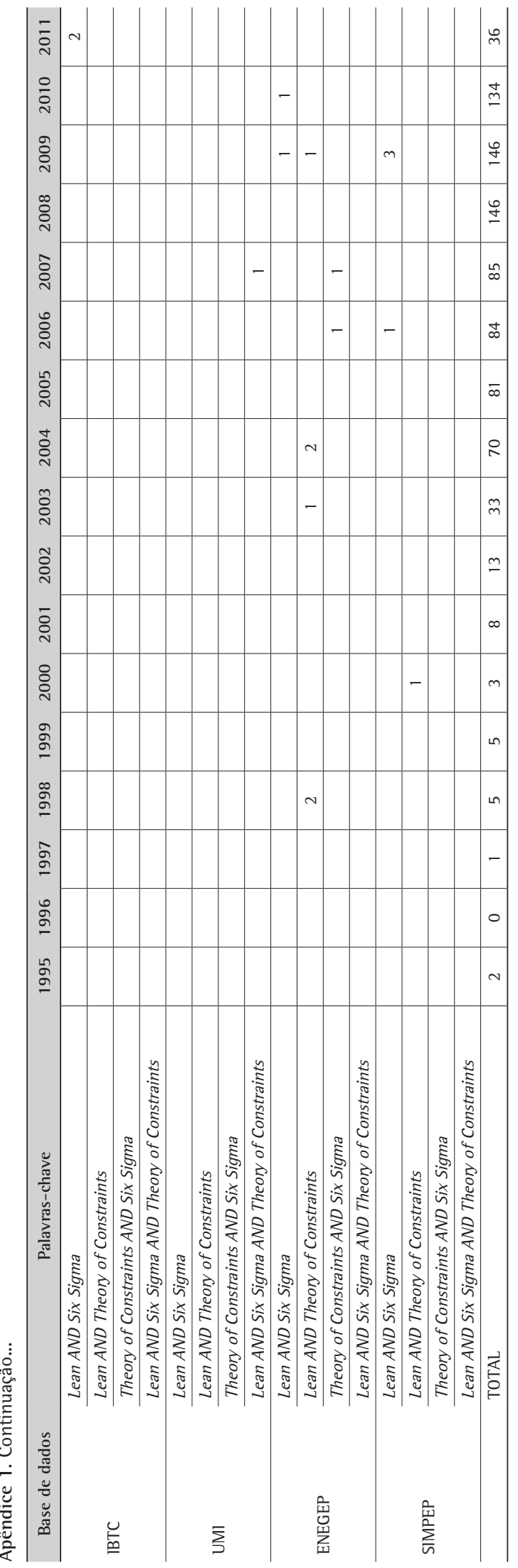

